An advant age of bi pedal humanoi d robot on the empat hy gener at i on: A neur oi magi ng st udy

\begin{tabular}{|c|c|}
\hline 著者 & $\begin{array}{l}\text { M ur a Naoki, Sugi ur a Mbt oaki, Takahashi } \\
\text { Nakot o, Nor i dai ra Tombhi sa, M yambt o At sushi, } \\
\text { Kur oki Yoshi hi r o, Kawashi ma Ryut a }\end{array}$ \\
\hline $\begin{array}{l}\text { j our nal or } \\
\text { publ i cat i on titl e }\end{array}$ & $\begin{array}{l}2008 \text { I EEE/RSJ I nt er nat i onal Conf er ence on } \\
\text { I nt el I i gent Robot s and Syst ens, I ROS }\end{array}$ \\
\hline page $r$ ange & $2465-2470$ \\
\hline year & 2008 \\
\hline URL & /hdl . handl e. net /10173/627 \\
\hline
\end{tabular}




\title{
An advantage of bipedal humanoid robot on the empathy generation: a neuroimaging study
}

\author{
Naoki Miura, Motoaki Sugiura, Makoto Takahashi, Tomohisa Moridaira, Atsushi Miyamoto, \\ Yoshihiro Kuroki, and Ryuta Kawashima
}

\begin{abstract}
To determine the effect of robotic embodiment on human-robot interaction, we used functional magnetic resonance imaging (fMRI) to measure brain activity during the observation of emotionally positive or neutral actions performed by bipedal or wheel-drive humanoid robots. fMRI data from 30 participants were analyzed in the study. The results revealed that bipedal humanoid robot performing emotionally positive actions induced the activation of the left orbitofrontal cortex, which is associated with emotional empathy, whereas wheel-drive humanoid robot performing the same actions elicited a lesser response. These results demonstrate that humans more readily empathize with a bipedal humanoid robot based on the ability to simulate human-like body movements.
\end{abstract}

\section{INTRODUCTION}

Recent advances in robots have produced several new types of robots, including humanoid robots with interactive functions for humans. It is generally believed that these robots will provide a valuable communicative presence in the home environment in the near future. However, robotic embodiment may be particularly important in robots designed to interact with human users. Because humans derive a considerable amount of information from body movements, it is possible that humans will interact more effectively with bipedal humanoid robots that can simulate human actions. This is in contrast to other robot designs, such as wheel-drive humanoid robots, which are able to move more effectively, but perhaps communicate less effectively for the lack of human-like movement. Thus, it is

Manuscript received February 22, 2008. This research was supported by JST/RISTEX, R\&D promotion scheme for regional proposals promoted by TAO, JST/CREST, a Grant-in Aid for Young Scientist (A) (KAKENHI 18680026) from the MEXT, and Research center for Language, Brain and Cognition, Graduate School of International Cultural Studies, Tohoku University.

N. Miura is with Department of Intelligence Mechanical Systems Engineering, Kochi University of Technology, 185 Miyanokuchi, Tosayamada-cho, Kami, Kochi, 782-8502 Japan. (Corresponding author, phone: +81-887-57-2314; e-mail: miura.naoki@kochi-tech.ac.jp).

N. Miura, M. Sugiura, and R. Kawashima are with Department of Functional Brain Imaging, Institute of Development, Aging and Cancer (IDAC), Tohoku University, 4-1 Seiryo-cho, Aoba-ku, Sendai, Miyagi, 980-8575 Japan

M. Sugiura is with Department of Cerebral Research, National Institute for Physiological Sciences, 38 Myodaijicho-Nishigonaka, Okazaki, Aichi, 444-8585 Japan.

M. Takahashi is with Graduate School of Engineering, Tohoku University, 6-6 Aoba, Aramaki, Aoba-ku, Sendai, Miyagi, 980-8579 Japan.

T. Moridaira, A. Miyamoto, and Y. Kuroki are with Information Technologies Laboratories, Sony Corporation, 5-1-12 Kitashinagawa, Shinagawa-ku, Tokyo, 141-0001 Japan. necessary to evaluate the cognitive mechanisms by which humans interpret the appearance and actions of robots designed for direct human-robot interaction.

As a result of rapid progress in neuroimaging technology, cognitive neuroscience has provided a great deal of information regarding the relationship between various cognitive functions and brain activity, and numerous researchers have examined the cognitive processes that govern social interaction between humans [1,2]. Body actions are particularly important in interpreting another's emotions [3], not only in human communications, but also in the case of interaction with non-human avatars [4].

Our long-term objective is to realize a more sophisticated human-robot interaction, through a profound understanding of brain activity. Here, we used functional magnetic resonance imaging (fMRI) to examine brain activity while observing robots performing various movements that carry a positive or neutral emotional connotation in humans. We prepared two types of humanoid robots, bipedal and wheel-drive, to compare the effect of robotic embodiment on cognitive processes related to an emotional interpretation of the robot's actions. For comparison, we also measured brain activity while observing a human performing the same actions to assess whether the same cortical network was used. We chose to present emotionally positive actions, because it was expected that such positive actions would clearly differentiate between brain activity related to humans versus robots, and between bipedal versus wheel-drive robots.

\section{RELATED WORK}

\section{A. Neuroscience Research}

Processes related to social cognition are an active research topic in neuroscience. The theory used to explain how cognitive function relates to understanding the state of another person, such as emotions or intentions, has been referred to as the "Theory of Mind" or "Mentalizing" $[1,2]$. The neural mechanisms underlying the ability to understand another's intentions from his or her body language has been studied based on the "Mirror-Neuron System" [5]. Based on these theories, previous studies have investigated the neural mechanisms related to empathy for facial expressions [6] and body movements [7], and have suggested that the ventrolateral prefrontal cortex contributes to this emotional empathetic process. Moreover, several studies have showed that it is possible to use virtual avatars as experimental 


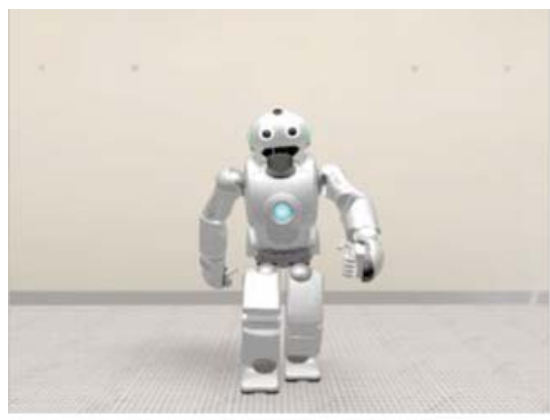

(a) Bipedal humanoid robot: QRIO

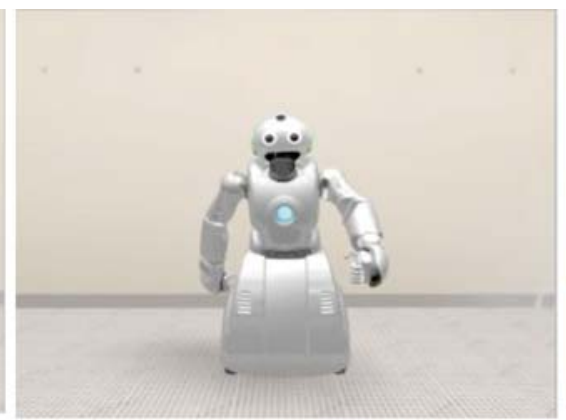

(b) Wheel-drive humanoid robot

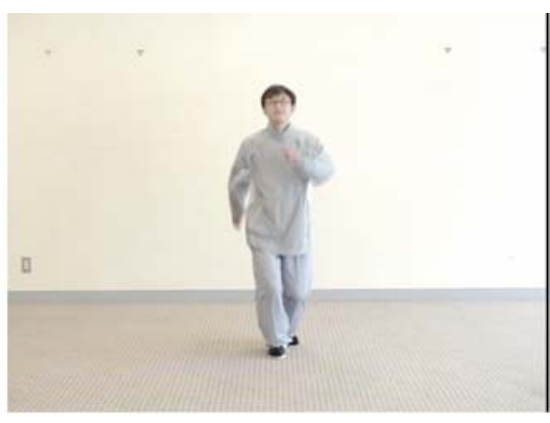

(c) Human actor (reference condition)

Fig. 1. Humanoid robots and human actor, which were used as experimental stimuli

stimuli for neuroimaging studies on social cognition $[8,9]$.

\section{B. Research on Robotics}

Although it is possible to build a human-like communicative robot, there are no clear criteria for human likeness. Recently, robotics research has focused on an integrated approach to robotics and cognitive science. Behavioral studies have described the relationship between the appearance of the robot and human affinity for it $[10,11]$, based on the "Uncanny Valley" hypothesis [12]; these studies suggest that affinity includes various factors, such as human likeness, familiarity, and eeriness. Although, motion is also an important, no clear explanation of the effect of motion has been established.

\section{EXPERIMENTAL HYPOTHESIS}

We examined two hypotheses in this experiment. First, we examined brain responses while observing robots perform various actions. We hypothesized that if humans feel that a robot is a communicative presence, then the cortical networks employed to process robot actions should be the same as those used while observing a human performing the same actions. Secondly, we hypothesized that if human-like embodiment is important to evoke emotional responses, then bipedal humanoid robots should elicit strong neurological responses than wheel-drive humanoid robots.

\section{RoBOt DESIGN}

\section{A. Bipedal and wheel-drive humanoid robots}

We used a small bipedal entertainment robot, QRIO (Sony Corporation, Tokyo, Japan; Fig. 1(a)) for this experiment. QRIO was developed to socially interact with humans in a home environment. It has 30 degrees of freedom (DoF) in its major joints, and each hand has five independent fingers. It can perform complex body movements through whole body cooperative dynamic motion control [13]. These movements are generated by the creative motion planning framework [14].

To analyze the effect of robotic embodiment on emotional processes, we created a wheel-drive humanoid robot (Fig. 1(b)). The upper part of the body was the same as the QRIO to minimize differences in visual and motion information. It had the same DoF in joints of the upper part of body as
QRIO, but it did not have leg joints. Thus, the characteristics of the motion that differentiate the bipedal from wheel-drive robot were the gait and having up-down motion of trunk part by flexure and extension of hip, knee and ankle joints.

\section{B. Emotional action by the robot}

Color video clips showing several emotionally positive and neutral actions performed by the bipedal and wheel-drive humanoid robots were created. Emotionally positive actions were created based on actions that elicit positive emotions in daily life, such as walking happily, pumping the arms, and raising both hands. Emotionally neutral actions were created in reference to positive actions and adjusted to not exhibit any emotional state. All video clips were filmed in an office setting. Twenty 3-s video clips were prepared for each of four conditions. Since the there was the difference of DoF on lower body joints between two robots, the action of the upper body part was the same, but differences appeared for the gait and having up-down motion. To examine cognitive mechanism during observing emotionally human actions, a professional actor was requested to repeat the aforementioned positive and neutral actions while maintaining a neutral facial expression (Fig. 1c). A digital camera was used to record 20 video clips in a similar setting. All clips were confirmed whether these were surely emotional or not by another 10 volunteers beforehand.

\section{EXPERIMENTAL METHODS}

\section{A. fMRI experimental environment}

Figure 2 shows our fMRI experimental environment (1.5

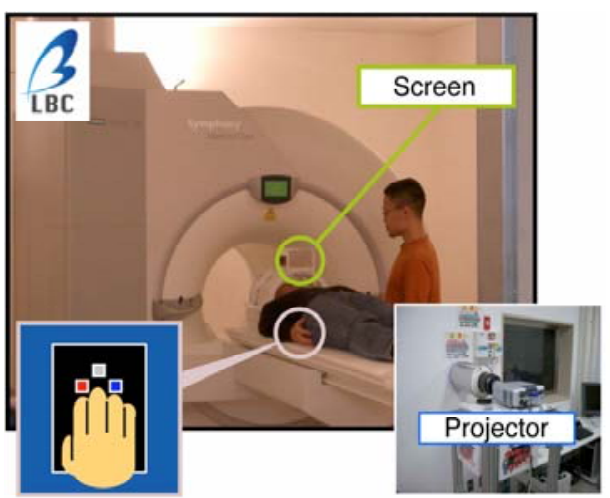

Fig. 2. fMRI experimental environment 
T Magnetom Symphony scanner; Siemens, Munich, Germany) at the Research Center for Language, Brain, and Cognition, Graduate School of International Cultural Studies, Tohoku University. The participant adopted a supine position inside the MRI scanner. A semi-lucent screen was positioned in front of the participant's face, and visual stimuli were projected from outside the MRI room. A three-button response pad was placed inside the chamber so that the participant could operate it comfortably with his or her right hand.

\section{B. Experimental Tasks}

The fMRI experiment was carried out with an event-related design of seven conditions. A timeline of the experiment is shown in Figure 3. The visual stimuli were presented using Presentation software (Neurobehavioral Systems, CA, USA).

Six experimental conditions were examined: emotionally positive actions by the bipedal (Bipedal-P) robot, the wheel-drive (Wheel-P) robot, or the human actor (Human-P), and emotionally neutral actions by the bipedal (Bipedal-N) robot, the wheel-drive (Wheel-N) robot, or the human actor (Human-N). Additionally, video clips of moving mosaic pictures (Mosaic) were also prepared to control for cognitive processes involved in low-order visual processing. In total, 140 video clips (20 clips $\times 7$ conditions) were presented during the fMRI measurement. One video clip was presented in each event and the order of the video clips was counterbalanced among the participants. Each inter-stimulus interval (ISI) was set at $1-8 \mathrm{~s}$, during which a fixationcross was presented at the center. Immediately before the first event and after the last event, the participants were permitted a 30 -s rest period while viewing the fixation cross. The duration of the entire fMRI scan was $15 \mathrm{~min}$.

\section{Instruction for participants}

During the fMRI scan, the participants were asked to indicate whether or not they felt a positive emotion toward the video clip by pressing the appropriate button with their right index or middle finger, respectively. Additionally, when the mosaic picture was presented, the participants were asked to press the button with their middle finger. In approximately half of the participants, finger assignment was reversed to control for brain activity associated with finger movement.

\section{D. fMRI data acquisition}

fMRI time series data covering the entire brain in 33 axial slices were acquired using gradient echo-echo planar imaging with a standard head coil (repetition time, $3000 \mathrm{~ms}$; echo time, $50 \mathrm{~ms}$; field-of-view, $192 \times 192 \mathrm{~mm}$; flip angle, 90 degrees; matrix size, $64 \times 64$; slice thickness, $3 \mathrm{~mm}$; inter-slice gap, $1 \mathrm{~mm}$; voxel size, $3 \times 3 \times 4 \mathrm{~mm}$ ). In addition, T1-weighted anatomical MR images were also acquired.

\section{E. Participants}

Thirty-nine right-handed healthy volunteers (26 males, 13 females; range, 19- 54 years old; mean, 24 years old) were enrolled in the study. Handedness was assessed using the Edinburgh Handedness Inventory [15]. The study was approved by the institutional review board at the Tohoku University School of Medicine and was in accordance with the Helsinki Declaration of Human Rights, 1975. Written informed consent was obtained from each participant.

\section{F. Data Processing and Statistical Analysis}

Data preprocessing and the statistical analysis of fMRI data were performed using Statistical Parametric Mapping 2 software (SPM2; Wellcome Department of Cognitive Neurology, London, UK [16]) running on MATLAB (MathWorks, MA, US). First, fMRI data were preprocessed to normalize the location of brain activity within the same anatomical space across all participants. The coordinates used for this analysis were set out by the Montreal Neurological Institute space (MNI space; origin, anterior commissure; $\mathrm{x}$-axis, right-left; $\mathrm{y}$-axis, anterior-posterior; z-axis, top-bottom).

\section{1) fMRI data preprocessing}

The initial five scans from each participant were dummy scans used to equilibrate the state of magnetization and were discarded from the data set. The effects of head motion across all scans were corrected by realigning to the first scan. Differences in acquisition timing among the 33 slices were adjusted to the 16 th slice. Data were spatially normalized to

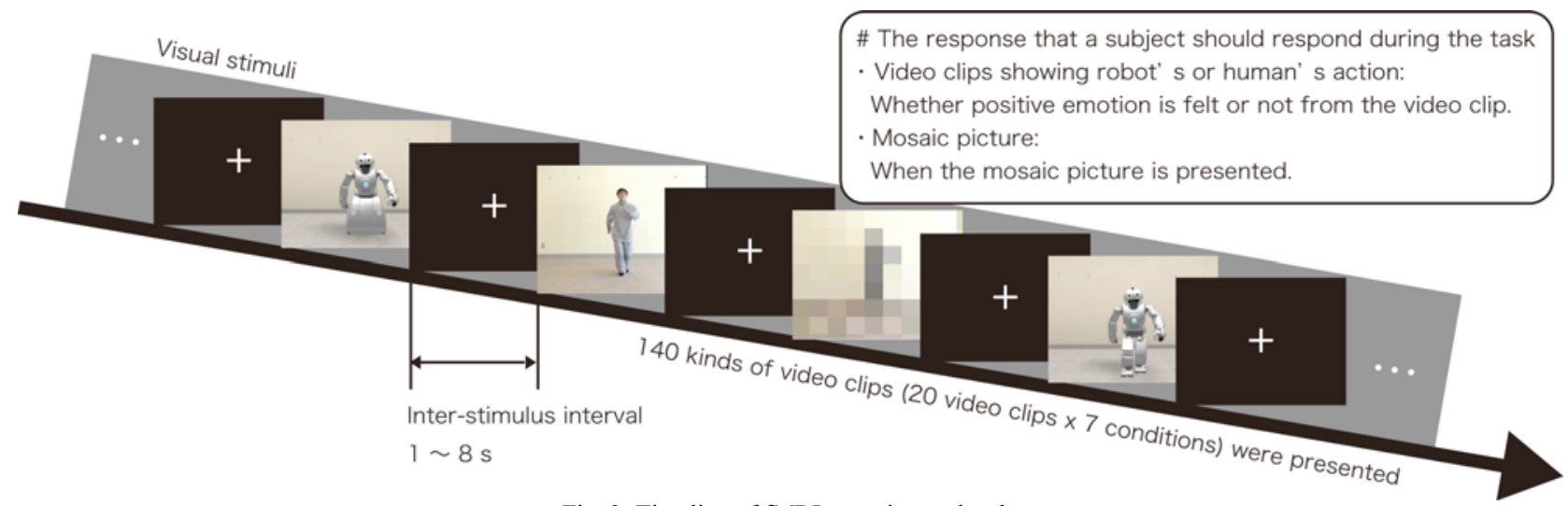

Fig. 3. Timeline of fMRI experimental task. 


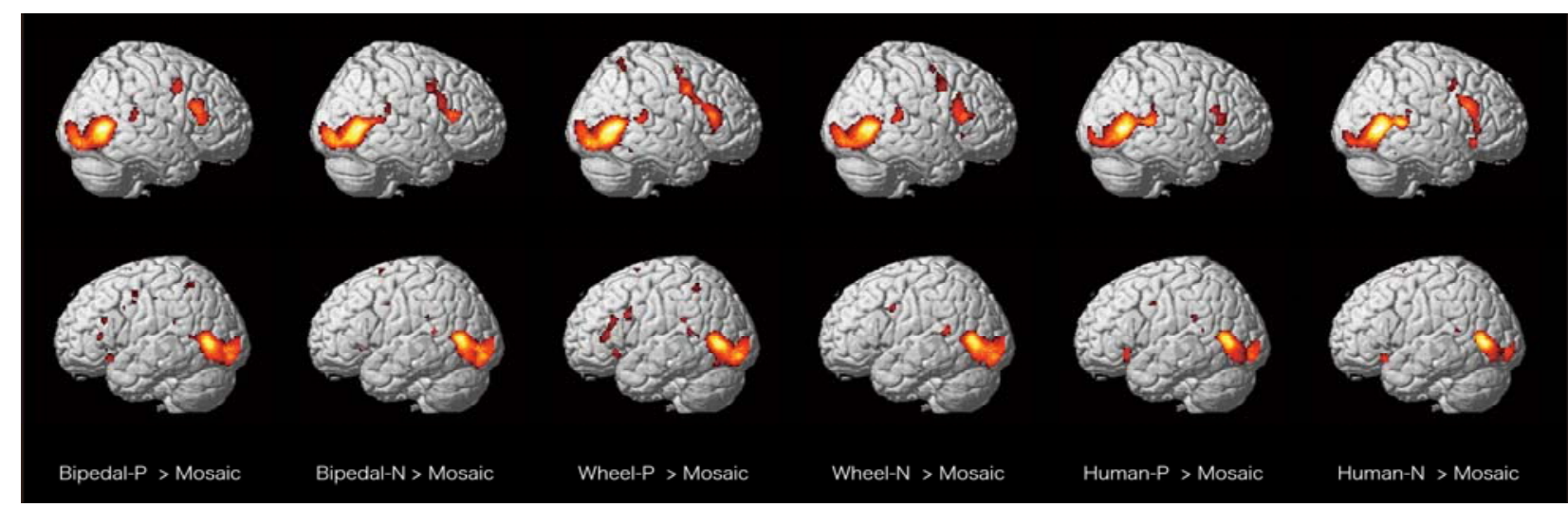

Fig. 4. Color-coded SPM results projected onto MNI anatomical template showing intersubject activation maps of the conventional subtraction analyses. The upper and lower rows show the right and left hemisphere that was projected activation areas, respectively.

the MNI-T1 template, using the anatomical MR image for each subject. Finally, each scan was smoothed with a 3D Gaussian filter ( $9 \mathrm{~mm}$ full-width at half-maximum) to reduce noise and minimize the effects of normalization errors.

Data from nine subjects were excluded because of their head movement (movement $<3 \mathrm{~mm}$ or rotation $<3$ degrees for each axis). Thus, our final data set included 30 participants (20 males, 10 females).

2) Conventional subtraction analysis

The fMRI data were statistically analyzed using a conventional two-stage approach in SPM2. First, the individual activation maps produced by each condition were assessed using a general linear model. For the analysis, each event was re-categorized as an emotionally positive or neutral condition according to the subjective selection on fMRI scanning by each subject. The experimental model hypothesized that hemodynamic responses would occur at each event (i.e., presentation of the Bipedal-P, Bipedal-N, Wheel-P, Wheel-N, Human-P, Human-N, or Mosaic clips). The onset of each event was set at the time that the participant pressed the button. Global signal changes were adjusted by proportional scaling, and low-frequency confounding effects were removed using a high-pass filter with a 256-s cutoff period. Multiple regression analyses were performed on each voxel to estimate the partial regression coefficient, showing that MR signal changes correlated with the hypothesized model.

We created subtraction images showing differences in brain activity between conditions. To identify brain activity related to observing body movement, we made subtraction of each action observation condition $>$ Mosaic condition (e.g. Bipedal-P > Mosaic), respectively. To identify brain activity related to an emotional interpretation of body movement, we compared response recorded during the following stimuli: Bipedal-P $>$ Bipedal-N and Wheel-P $>$ Wheel-N.

Second, intersubject activation maps were created by performing a one-sample t-test on each voxel from each subtraction image. Furthermore, in the cases of Bipedal-P > Bipedal-N and Wheel-P $>$ Wheel-N, the activation maps for Bipedal-P > Mosaic and Wheel-P > Mosaic (statistical threshold, $\mathrm{P}<0.05$ ), respectively, were used as mask images to remove voxels that did not reach the level of significance in each comparison. The level of statistical significance was set at $\mathrm{P}<0.05$ and corrected for multiple comparisons using family-wise error.

3) Region of interest analysis

To evaluate the effect of robotic embodiment on cognitive processes related to emotional interpretation, a region of interest (ROI) analysis was performed to inspect local changes in the hemodynamic response of the observed activation peaks. We defined the peak location in the activated clusters within the Bipedal-P $>$ Bipedal-N and Wheel-P > Wheel-N contrasts as ROIs, and the parameter estimates, that mean the estimated partial regression coefficients, of each condition were extracted. We compared the differences between the parameter estimates for Bipedal-P > Bipedal-N and Wheel-P > Wheel-N on ROIs.

\section{RESULTS}

\section{A. Conventional subtraction analysis}

Figure 4 shows activation maps of each of the six test conditions compared to the Mosaic condition. From the results, common activation patterns were observed in the bilateral occipito-temporal junction extending to superior temporal sulcus, right ventral premotor area extending to inferior frontal gyrus, and supplementary motor area.

Activated areas related to interpreting emotionally positive actions are summarized in Figure 5. In the case of Bipedal-P > Bipedal-N, significant activation was observed in the left orbitofrontal cortex. In the case of Wheel-P > Wheel-N, activation clusters were observed in the bilateral occipito-temporal junction area, right fusiform and middle temporal gyri, and right midbrain.

\section{B. Region of interest analysis}

Figure 6 shows the average parameter estimates for each condition in eight ROIs. The parameter estimate for Bipedal-P $>$ Bipedal-N was significantly greater than that for Wheel-P $>$ Wheel-N in the ROI of the left orbitofrontal gyrus. In contrast, in the case of four ROIs identified at the right occipito-temporal junction, right fusiform gyrus, right middle temporal gyrus, and right midbrain, the parameter estimate for Wheel-P > Wheel-N was significantly greater 

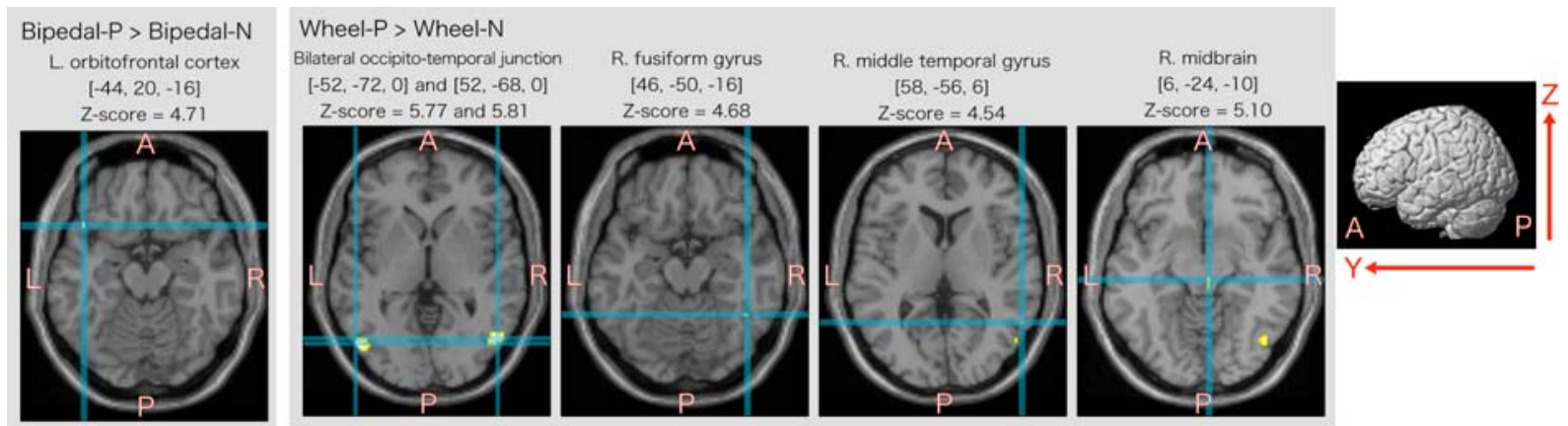

Fig. 5. Activated areas that specifically related to observation of emotionally positive action compared with neutral action. The activation areas were projected to axial slice (x-y plane) of MNI anatomical template.

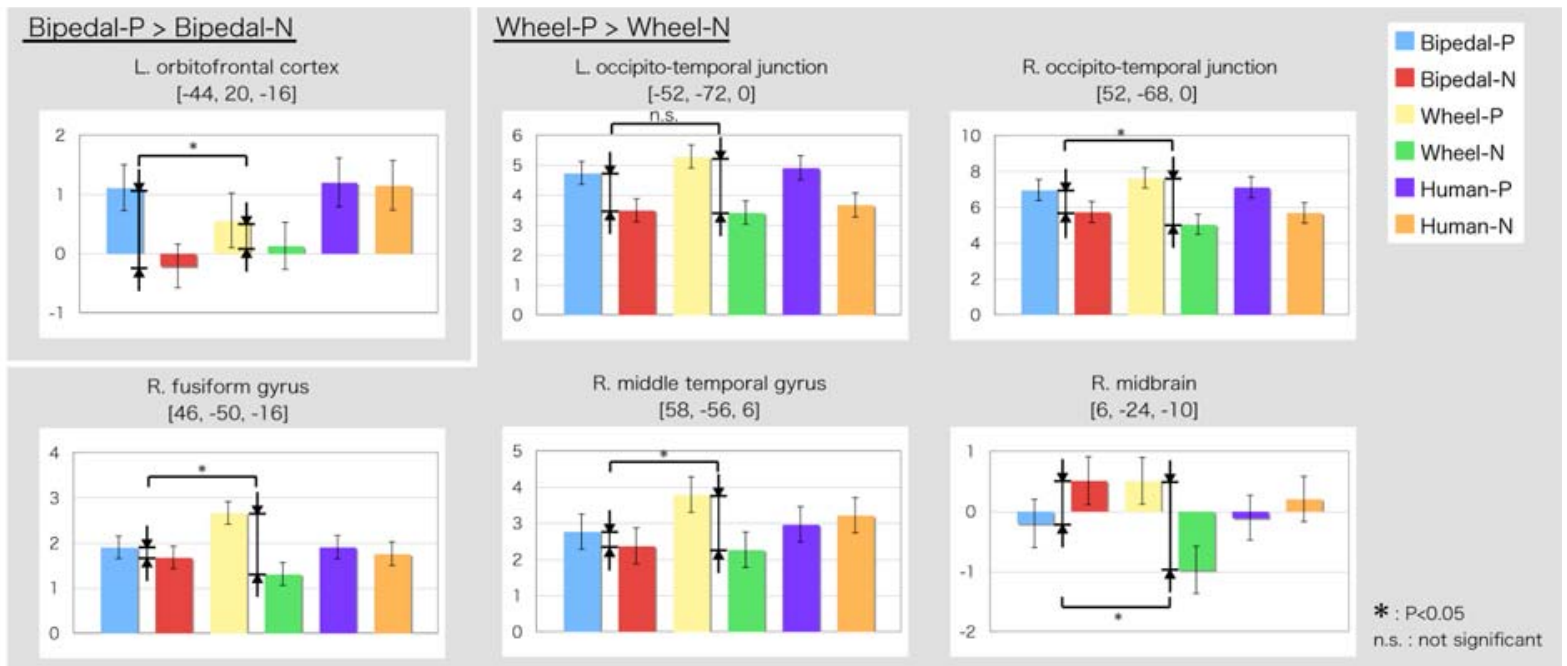

Fig. 6. Parameter estimates of action observation conditions on each ROI, and the statistical significance of difference between "Bipedal-P > Bipedal-N" vs. "Wheel-P > Wheel-N" contrasts. Error bar shows standard error of mean.

than that for Bipedal-P $>$ Bipedal-N.

\section{DISCUSSION}

\section{A. Perception of emotionally positive actions by the bipedal humanoid robot}

When the participants observed emotionally positive actions performed by a bipedal humanoid robot, the left orbitofrontal cortex was significantly activated. Although, there seems to be activation peak in the border of left orbitofrontal cortex and temporal pole, when we observed the activation using lower threshold, the activation cluster expanded toward the orbitofrontal cortex. Furthermore, the difference in local regional activity when comparing Bipedal-P > Bipedal-N was significantly larger than that when comparing Wheel-P $>$ Wheel-N. The orbitofrontal cortex and its adjacent areas are strongly associated with emotional processes $[17,18]$. A previous study also suggested that activity in this region is related to empathetic processing of emotional facial expressions shown by others [6] or painful stimuli for others [19], and to the interpretation of expressive gestures [7]. Our results suggest that, based on body movements alone, humans empathize more strongly to the "emotional state" of a bipedal humanoid robot than a wheel-drive humanoid robot.
Our two robots differed not only in appearance, but also in gait, which may have contributed to differences in emotional response. Because up-and-down motion of the trunk is an important element when positive emotions are expressed physically, the bipedal humanoid robot may have been able to communicate more effectively. Thus, when we evaluate human affinity for a communicative gesture by a robot, it is necessary to consider not only the simplicity of the gestures but also the human-likeness of the action, such as the up-and-down motion of the trunk.

We obtained approximately the same values for parameter estimates for the Human-P and Human-N conditions as the Bipedal-P condition in the left orbitofrontal cortex. Post-fMRI participant interviews revealed that they detected incongruities in the video clips, specifically because the actor performed emotional actions without any facial expression. Thus, although the participants were still capable of identifying positive actions, it is possible that that the local activity elicited by the Human-P condition in left orbitofrontal cortex may have been decreased by this incongruity between facial and bodily expression. When observing robots, the participants concentrated on the body of each robot, rather than the face, because they recognized that all robot faces were the same. Thus, the same incongruity may not have affected the perception of 
emotionally significant actions performed by the robot.

\section{B. Common activities during the action observation}

While observing each condition, common activation areas were identified in several brain areas compared to the mosaic condition. A previous study suggested that activity in the occipito-temporal junction was related to the visual processing of motion, and its boundary areas were related to the perception of biological motion [20]. Activity in the superior temporal sulcus may also contribute to social perception [2,7]. Furthermore, previous studies on action perception suggested that activity in the ventral part of the prefrontal area during the observation of body movements was reflected in the cognitive processes of understanding actions, as explained by the "Mirror Neuron System" [5]. Thus, our results reveal an activation pattern or cortical network related to the interpretation of body movements. More importantly, this pattern was activated while observing humanoid robots perform emotionally.

In the case of four ROIs obtained when comparing Wheel-P $>$ Wheel-N in the right hemisphere, the difference in parameter estimates for Wheel-P $>$ Wheel-N was significantly larger than that for Bipedal-P $>$ Bipedal-N. It is possible that the appearance of a wheel-drive humanoid robot induced additional processing, which was required for the interpretation of emotional actions within the cortical network for action understanding.

\section{Contribution to human-robot interactions and future work}

To further robotic technology, it is critical to understand the interaction between humans and robots. For this reason, it is necessary to determine what information is being processed in the human brain while viewing robots and their movements. Here, we used cognitive neuroscience to demonstrate the advantage of bipedal humanoid robots in communicative applications. More specifically, we developed a method to examine the details of information processing during human-robot interactions and provided fundamental evidence using fMRI. Similar research concept was indicated by previous robotics studies [10], and our directionality will contribute to future robotics research.

The present study has some limitations. Because of the physical limitations of fMRI, we were unable to measure brain activity related to direct interaction, such as touching the robot or sharing a common environment. However, other neuroimaging methods, such as near-infrared spectroscopy (NIRS), may be useful in this respect. Second, although we focused on the effect of robotic embodiment, future studies will be required to evaluate longitudinal changes in emotional affinity as a result of human-robot interaction.

\section{CONCLUSIONS}

Our results demonstrate that bipedal humanoid robots elicit a higher degree of activation in the left orbitofrontal cortex compared to wheel-drive humanoid robots performing the same emotionally positive actions. Because this region of the brain is related to empathetic processes, humans more readily empathize with a bipedal humanoid robot. Therefore, our results indicate the advantage of bipedal humanoid robots in communicative applications.

\section{ACKNOWLEDGEMENT}

We would like to thank our colleagues at Department of Functional brain Imaging, IDAC, Tohoku University for their help on this experiment.

\section{REFERENCES}

[1] D.M. Amodio, and C.D. Frith, "Meeting of minds: the medial frontal cortex and social cognition," Nat. Rev. Neurosci., 7, 268-277. 2007.

[2] M.D. Lieberman. "Social cognitive neuroscience: a review of core process," Annual Review of Psychology. 58, 259-89. 2007.

[3] B. de Gelder, "Towards the neurobiology of emotional body language," Nat. Rev. Neurosci., 7, 242-249. 2006.

[4] N. Bianchi-Berthouze, A. Kleinsmith, "A categorical approach to affective gesture recognition," Connection Science, 15(4), 259 - 269, 2003

[5] G. Rizzolatti, L. Fogassi, and V. Gallese, "Neurophysiological mechanisms underlying the understanding and imitation of action," Nat. Rev. Neurosci., 2, 661-669. 2001.

[6] M. Jabbi, M.Swart, and C. Keysers, "Empathy for positive and negative emotions in the gustatory cortex," NeuroImage, 34. 1744-1753. 2007

[7] M. Lotze, U. Heymans, N. Birbaumer, R. Veit, M. Erb, R. Flor, and U. Halsband, "Differential cerebral activation during observation of expressive gestures and motor acts," Neuropsychologia, 44, 1787-1795. 2005.

[8] E. Moser, B. Derntl, S. Robinson, D. Fink, R.C. Gur, and L. Grammer, "Amygdala activation at $3 \mathrm{~T}$ in response to human and avatar facial expressions of emotions," Journal of Neuroscience Methods. 161(1), 126-133. 2007.

[9] L. Schilbach, A.M. Wohlschlaeger, N.C. Kraemer, A. Newen, N.J. Shah, G.R. Fink, and K. Vogeley, "Being with virtual others: Neural correlates of social interaction," Neuropsychologia. 44. 718-730. 2006.

[10] K.F. MacDorman, and H. Ishiguro, "The uncanny advantage of using androids in cognitive science research," Interaction Studies, 7(3), 297-337. 2006.

[11] K.F. MacDorman, "Subjective ratings of robot video clips for human likeness, familiarity and eeriness: and exploration of the uncanny valley," ICCS/CogSci-2006 Long Symposium: Toward Social Mechanisms of Android Science.

[12] M. Mori, "Bukimi no tani [the uncanny valley]," Energy, 7(4), 33-35. 1970.

[13] Y. Kuroki, T. Ishida, J. Yamaguchi, and K. Nagasaka, "Small biped walking entertainment robot SDR-4X with a highly integrated motion control," In Proceedings of the 20th Annual Conference on RSJ. Osaka, Japan, 1C17, 2002.

[14] T. Moridaira, A. Miyamoto, S. Shimizu, Y. Kawanami, K. Nagasaka, and Y. Kuroki, "The interactive motion control for a small humanoid robot," In Proceedings of the 5th IEEE-RAS International Conference on Humanoid robots. 463-468, 2005.

[15] R. Oldfield, "The assessment and analysis of handedness: The Edinburgh inventory," Neuropsychologia. 9, 812-815. 1971.

[16] R.S.J. Frackowiak, K.J. Friston, C. Frith, R. Dolan, C.J. Price, S. Zeki, J. Ashburner, and W.D. Penny, Human Brain Function. Academic Press, 2nd edition, 2003

[17] J.D. Steele, and S.M. Lawrie, "Segregation of cognitive and emotional function in the prefrontal cortex; a streotactic meta-analysis," Neuroimage, 21, 868-875. 2004.

[18] N.L Rempel-Clower,"Role of orbitofrontal cortex connections in emotion," Ann N Y Acad Sci. 1121:72-86, 2007.

[19] T. Singer, B. Seymour, J. O’Doherty, H. Kaube, R.J. Doran, C.D. Frith, "Empathy for pain involves the affective but not sensory component for pain," Science, 303, 1157-1162, 2004.

[20] M.A. Giese, and T. Poggio, "Neural mechanisms for the recognition of biological movement," Nat Rev Neurosci. 4(3), 179-92. 2003 